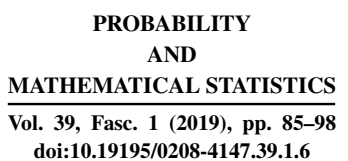

\title{
ASYMMETRICALLY TEMPERED STABLE DISTRIBUTIONS WITH APPLICATIONS TO FINANCE
}

\author{
BY
}

\author{
A. A REF I (Allameh Tabataba'i University)
}

AND R. POURTA HER I (Allameh TABATABA' I University)

Abstract. In this paper, we introduce a technique to produce a new family of tempered stable distributions. We call this family asymmetrically tempered stable distributions. We provide two examples of this family named asymmetrically classical modified tempered stable (ACMTS) and asymmetrically modified classical tempered stable (AMCTS) distributions. Since the tempered stable distributions are infinitely divisible, Lévy processes can be induced by the ACMTS and AMCTS distributions. The properties of these distributions will be discussed along with the advantages in applying them to financial modeling. Furthermore, we develop exponential Lévy models for them. To demonstrate the advantages of the exponential Lévy ACMTS and AMCTS models, we estimate parameters for the S\&P 500 Index.

2010 AMS Mathematics Subject Classification: Primary: 60E07; Secondary: 91B84.

Key words and phrases: Tempered stable distributions, exponential Lévy model.

\section{INTRODUCTION}

Black and Scholes [3] used the normal distribution for the pricing and hedging theory for the option market. This model cannot describe the skewed and fattailed properties of the empirical distribution of asset returns. Hurst et al. [9] applied the $\alpha$-stable distribution in modeling financial asset returns because it allows for skewness and fat tails. Empirical studies showed that asset returns time series present heavier tails than the normal distribution and thinner tails than the $\alpha$-stable distribution. To overcome the drawbacks of the $\alpha$-stable distribution, the class of tempered stable distributions has been introduced by Koponen [16], Boyarchenko and Levendorskii [4] and Carr et al. [5]. These distributions, sometimes called the classical tempered stable (CTS) distributions, have not only heavier tails than the normal distribution and thinner than the $\alpha$-stable distribution but also have finite 
moments of all orders. Barndorff-Nielsen and Levendorskii [ [1] originally obtained a time-changed Brownian motion using a tempered stable subordinator. The modified tempered stable (MTS) distribution was introduced by Kim et al. [15]. Also, Kim et al. [14] defined the normal tempered stable (NTS) distribution by the exponential tilting for the symmetric MTS distribution. The rapidly decreasing tempered stable (RDTS) distribution was introduced by Bianchi et al. [2] and Kim et al. [12]. Rosiński [19] characterized the tempered stable process by the spectral measure. Rosiński and Sinclair [20] introduced generalized tempered stable (GTS) distributions. Kim et al. [10] presented market models based on the tempered stable distributions and provided empirical tests of these distributions. Küchlera and Tappe [17] reviewed tempered stable distributions and provided some of their basic properties.

The tempered stable distributions are constructed by taking an $\alpha$-stable distribution with $\alpha \in(0,2)$ and multiplying the Lévy measure by a function onto each half of the real axis. For example, this function for the CTS distribution is decreasing exponentially and for the MTS distribution is a modified Bessel function of the second kind. However, the tempered stable distributions explain the observed market returns better than the $\alpha$-stable distribution, which still does not fit to these data sufficiently well.

More specifically, in stock markets, the returns are usually moved by news. A piece of good news increases stock prices, yet some of this increase gets dampened by the increase in risk premium requested for the higher volatility. On the other hand, a piece of bad news lowers stock prices. This drop gets further amplified by the increase in the risk premium. Because of the clustering of news, the left tail of returns' distribution of stocks could therefore be different from the right tail. In this paper, we introduce a new family of distributions which allows us to use a tempering function for each side of the real axis to capture the different behavior tails. They have a TS distribution feature and we call them asymmetrically tempered stable (ATS) distributions. An asymmetrically tempered stable distribution is a combination of two TS distributions. For example, if we use a decreasing exponential function for the negative real axis and a modified Bessel function of the second kind for the positive real axis, the resulting distribution will be an asymmetrically classical modified tempered stable (ACMTS) distribution. Also, we can use a modified Bessel function of the second kind for the negative real axis and a decreasing exponential function for the positive real axis. In this case the resulting distribution is an asymmetrically modified classical tempered stable (AMCTS) distribution. The tempered stable distribution controls the skewness and kurtosis using at least four parameters. Empirically, we show that the ATS distributions are able to provide a good fit to data with fewer parameters than tempered stable distributions.

In the Black-Scholes model [3], the stock price process is described by the exponential of Brownian motion. Replacing the driving process by a Lévy process, we obtain the class of exponential Lévy models. For example, the exponen- 
tial ACMTS and AMCTS models can be obtaind by using ACMTS and AMCTS processes as the driving processes. The main problem with the exponential Lévy models is that they generate an incomplete market, that is, the equivalent martingale measure (EMM) of a given market measure is not unique in general. For this reason, we need a method to select one reasonable EMM in the incomplete market generated by an exponential Lévy model. We use the Esscher transform presented by Gerber and Shiu [7], [8] to select an EMM.

The rest of this paper is organized as follows. In Section 2, we review the CTS, MTS and GTS distributions. In Section 3, we introduce ACMTS and AMCTS distributions and present some of their basic properties. The exponential ACMTS and AMCTS market models are discussed in Section 4. We will show the estimation results for the market parameters for the historical distribution of the log-returns of the S\&P 500 Index in Section 5.

\section{TEMPERED STABLE DISTRIBUTIONS}

In this section, we review the definitions of the classes of the tempered stable distributions introduced by Rosiński [19] and Rosiński and Sinclair [20]. The polar coordinates representation of a measure $\lambda=\lambda(d x)$ on $\mathbb{R}_{0}^{d}:=\mathbb{R}^{d} \backslash\{0\}$ is the measure $\lambda=\lambda(d r, d u)$ on $(0, \infty) \times S^{d-1}$ obtained by the bijection $x \mapsto\left(\|x\|, \frac{x}{\|x\|}\right)$. Let the Lévy measure $\nu$ of an $\alpha$-stable distribution on $\mathbb{R}^{d}$ in polar coordinates be of the form

$$
\nu(d r, d u)=\alpha r^{-\alpha-1} d r \sigma(d u),
$$

where $\alpha \in(0,2)$ and $\sigma$ is a finite measure on $S^{d-1}$. A tempered $\alpha$-stable distribution is defined by tempering the radial term of $\nu$ as follows:

DEFINITION 2.1 (Rosiński [II9]). Let $\alpha \in(0,2)$ and $\sigma$ be a finite measure on $S^{d-1}$. A probability measure $\mu$ is called tempered stable (TS) if it is infinitely divisible without Gaussian part whose Lévy measure $\nu$ can be written in polar coordinates as

$$
\nu(d r, d u)=\alpha r^{-\alpha-1} q(r, u) d r \sigma(d u),
$$

where $q(\cdot, u):(0, \infty) \times S^{d-1} \mapsto(0, \infty)$ is a completely monotone Borel function with $q(\infty, u)=0$ for each $u \in S^{d-1}$. The complete monotonicity of $q(\cdot, u)$ means that $(-1)^{n} \frac{d^{n}}{d r^{n}} q(r, u)>0$ for all $r>0, u \in S^{d-1}$ and $n=0,1,2, \ldots$ In particular, $q(\cdot, u)$ is strictly decreasing and convex. The function $q$ is called a tempering function.

DEFINITION 2.2 (Rosiński and Sinclair [20]). An infinitely divisible distribution $\mu$ on $\mathbb{R}^{d}$ is said to be generalized tempered stable (GTS) if $\mu$ has no Gaussian part and the Lévy measure $\nu$ of $\mu$ can be represented as

$$
\nu(B)=\int_{S^{d-1}} \int_{0}^{\infty} 1_{B}(r u) q(r, u) r^{-\alpha-1} d r \sigma(d u), \quad B \in \mathcal{B}\left(\mathbb{R}^{d}\right),
$$


where $\alpha \in(0,2), \sigma$ is a finite measure on $S^{d-1}$, and $q:(0, \infty) \times S^{d-1} \mapsto \mathbb{R}_{+}$is a measurable function such that for some nonnegative function $g \in L^{1}\left(S^{d-1}, \sigma\right)$

$$
\lim _{r \mapsto 0}\|q(r, \cdot)-g(\cdot)\|_{L^{1}\left(S^{d-1}, \sigma\right)}=0 .
$$

Moreover, Rosiński and Sinclair [20] present the characteristic function, the cumulant function, short- and long-time behavior, absolute continuity, and shot-noise type series representation for GTS distributions and Lévy processes induced by the GTS distributions.

2.1. Classical tempered stable distribution. In this subsection, we review classical tempered stable (CTS) distributions which were introduced under different names, including: the truncated Lévy flight (Koponen [16]), the KoBoL distribution (Boyarchenko and Levendorskii [4]), and the CGMY (Carr et al. [5]). Rosiński [19] and Kim et al. [14] define the CTS distribution as follows:

Definition 2.3. Let $C, \lambda_{+}, \lambda_{-}>0, \alpha \in(0,2)$, and $\mu \in \mathbb{R}$. An infinitely divisible distribution is a CTS distribution with parameters $\left(\alpha, C, \lambda_{+}, \lambda_{-}, \mu\right)$ if its Lévy triplet $(A, \nu, \gamma)$ is given by $A=0$ and

$$
\nu(d x)=\left(\frac{C e^{-\lambda_{+} x}}{x^{\alpha+1}} 1_{x>0}+\frac{C e^{-\lambda_{-}|x|}}{|x|^{\alpha+1}} 1_{x<0}\right) d x,
$$

and

$$
\gamma=\mu-\int_{|x|>1} x \nu(d x) .
$$

If a random variable $X$ follows the CTS distribution, then we write $X \sim$ $C T S\left(\alpha, C, \lambda_{+}, \lambda_{-}, \mu\right)$. A Lévy process induced from the CTS distribution is called a CTS process with parameters $\left(\alpha, C, \lambda_{+}, \lambda_{-}, \mu\right)$. The Lévy measure of the CTS distribution in polar coordinates can be written as

$$
\nu(d r, d u)=r^{-\alpha-1} q(r, u) d r \sigma(d u),
$$

where $q(r, 1)=e^{-\lambda_{+} r}, q(r,-1)=e^{-\lambda_{-} r}$ and $\sigma(1)=\sigma(-1)=C$. The function $q(\cdot, u)$ is a completely monotone function on $(0, \infty)$ satisfying $q(\infty, u)=0$ and $q\left(0_{+}, u\right)=1$. In particular, $q(\cdot, u)$ is strictly decreasing, convex, and bounded by one. The condition (2.2) holds with $g(u)=1$, so the CST distribution belongs to the class of TS and GTS distributions. The characteristic function $\phi_{C T S}$ for a CTS distribution is given by

$$
\phi_{C T S}(u)=\exp \left(i u m+G_{C}\left(i u ; \alpha, \lambda_{+}, C\right)+G_{C}\left(-i u ; \alpha, \lambda_{-}, C\right)\right),
$$

where

$$
G_{C}(x ; \alpha, \lambda, C)=C \Gamma(-\alpha)\left((\lambda-x)^{\alpha}-\lambda^{\alpha}\right)-x C \Gamma(1-\alpha) \lambda^{\alpha-1}
$$

for some $x \in \mathbb{R}$. The proof was given by Carr et al. [5] and Cont and Tankov [6]. 
2.2. The MTS distribution. In this subsection, we review the MTS distribution which was introduced by Kim et al. [1]].

DEFINITION 2.4. An infinitely divisible distribution is called an MTS distribution with parameters $\left(\alpha, C, \lambda_{+}, \lambda_{-}, \mu\right)$ if its Lévy triplet $(A, \nu, \gamma)$ is given by $A=0$ and

$$
\nu(d x)=C\left(\frac{\lambda_{+}^{(\alpha+1) / 2} K_{(\alpha+1) / 2}\left(\lambda_{+} x\right)}{x^{(\alpha+1) / 2}} 1_{x>0}+\frac{\lambda_{-}^{(\alpha+1) / 2} K_{(\alpha+1) / 2}\left(\lambda_{-}|x|\right)}{|x|^{(\alpha+1) / 2}} 1_{x<0}\right),
$$

and

$$
\gamma=\mu-\int_{|x|>1} x \nu(d x)
$$

where $C>0, \lambda_{+}, \lambda_{-}>0, \mu \in \mathbb{R}, \alpha \in(0,2) \backslash\{1\}, K_{p}(x)$ is the modified Bessel function of the second kind and $\sigma(1)=\sigma(-1)=C$.

The tempering function $q$ is of the form

$$
q(r, u)= \begin{cases}\left(\lambda_{+} r\right)^{(\alpha+1) / 2} K_{(\alpha+1) / 2}\left(\lambda_{+} r\right), & u=1, \\ \left(\lambda_{-} r\right)^{(\alpha+1) / 2} K_{(\alpha+1) / 2}\left(\lambda_{-} r\right), & u=-1 .\end{cases}
$$

DEFINITION 2.5. The radial tempering function $r \rightarrow q(r, u)$ is decreasing with

(2.4) $q(r, u) \rightarrow 1$ as $r \downarrow 0 \quad$ and $\quad q(r, u) \rightarrow\left(\frac{\pi}{2}\right)^{1 / 2}\left(\lambda_{ \pm} r\right)^{\alpha / 2} e^{-\lambda_{ \pm} r}$ as $r \uparrow \infty$.

It can be shown that $q(\cdot, u)$ is not completely monotone. Thus the MTS distributions are not included in the class of TS distributions but belong to the class of GTS distributions with $g(u)=1$ in (2.2).

The characteristic function $\phi_{M T S}$ for a modified tempered stable distribution is given by $\phi_{M T S}(u)=\exp \left(i u \mu+G_{M}\left(i u ; \alpha, \lambda_{+}, C\right)+G_{M}\left(-i u ; \alpha, \lambda_{-}, C\right)\right)$, where for $x \in \mathbb{R}$

$$
\begin{aligned}
G_{M}(x ; \alpha, \lambda, C)= & \frac{\sqrt{\pi} C \Gamma(-\alpha / 2)}{2^{(\alpha+3) / 2}}\left(\left(\lambda^{2}-x^{2}\right)^{\alpha / 2}-\lambda^{\alpha}\right) \\
& +\frac{x C \Gamma((1-\alpha) / 2)}{2^{(\alpha+1) / 2}} \lambda^{\alpha-1}\left(F\left(1, \frac{1-\alpha}{2} ; \frac{3}{2} ; \frac{x^{2}}{\lambda^{2}}\right)-1\right),
\end{aligned}
$$

and $F$ is the hypergeometric function. The proof can be found in Kim et al. [II] or Kim et al. [14]. If a random variable $X$ follows the MTS distribution, then we write $X \sim \operatorname{MTS}\left(\alpha, C, \lambda_{+}, \lambda_{-}, \mu\right)$. 


\section{ASYMMETRICALLY TEMPERED STABLE DISTRIBUTIONS}

In this section, we present two new tempered stable distributions by combining CTS and MTS distributions. We refer to them as the ACMTS and AMCTS distributions. In fact, we use a tempering function for each side of the real axis. The definitions and properties of these distributions are presented in the following.

\subsection{The ACMTS distribution.}

DEFINITION 3.1. An infinitely divisible distribution is called an ACMTS distribution with parameters $(\alpha, C, \lambda, \mu)$ if its Lévy triplet $(A, \nu, \gamma)$ is given by $A=0$ and

$$
\nu(d x)=\left(C \frac{e^{-\lambda|x|}}{|x|^{\alpha+1}} 1_{x<0}+C \frac{\lambda^{(\alpha+1) / 2} K_{(\alpha+1) / 2}(\lambda x)}{x^{(\alpha+1) / 2}} 1_{x>0}\right) d x
$$

and

$$
\gamma=\mu-\int_{|x|>1} x \nu(d x)
$$

where $C, \lambda>0, \mu \in \mathbb{R}$ and $\alpha \in(0,2) \backslash\{1\}$. If a random variable $X$ follows the ACMTS distribution, then we write $X \sim A C M T S(\alpha, C, \lambda, \mu)$.

The Lévy measure of the ACMTS distribution can be written in polar coordinates as

$$
\nu(d r, d u)=r^{-\alpha-1} q(r, u) d r \sigma(d u),
$$

where $q(r, 1)=(\lambda r)^{(\alpha+1) / 2} K_{(\alpha+1) / 2}(\lambda r), q(r,-1)=e^{-\lambda r}$. We can show that $q(\cdot, u)$ is decreasing and bounded by $g(u)=1$. Thus the ACMTS distributions are not included in the class of TS distributions but belong to the class of GTS distributions with $g(u)=1$ in (‥2). The characteristic function of the ACMTS distribution is given in the following:

THEOREM 3.1. Let $X \sim A C M T S(\alpha, C, \lambda, \mu)$. Then the characteristic function of $X$ is given by

$$
\phi_{X}(u)=\exp \left(i u \mu+G_{M}(i u ; \alpha, \lambda, C)+G_{C}(-i u ; \alpha, \lambda, C)\right) \text {, }
$$

where $G_{C}(u ; \alpha, \lambda, C)$ and $G_{M}(u ; \alpha, \lambda, C)$ are given by (2.3) and (2.5)), respectively. 


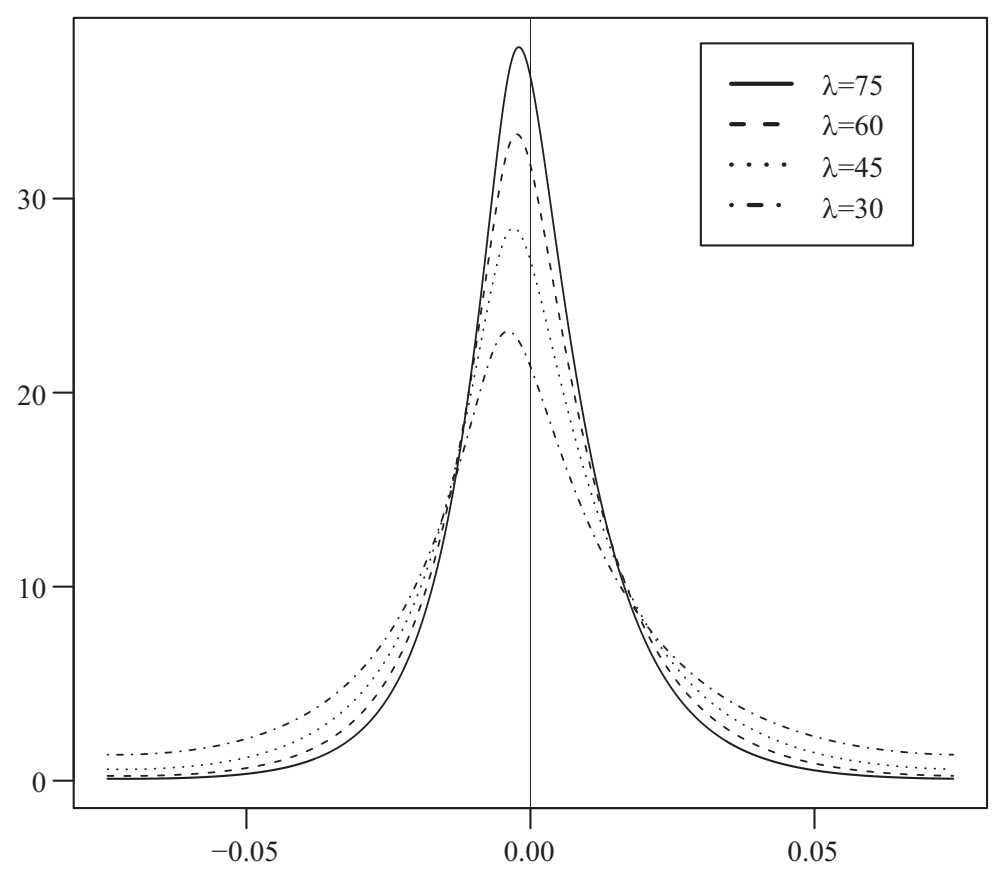

FIGURE 1. Probability density of the ACMTS distributions: dependence on $\lambda$. Parameters: $\mu=0, \alpha=0.5, C=0.07$.

Pro of. We have

$$
\begin{aligned}
& \int_{-\infty}^{\infty}\left(e^{i u x}-1-i u x 1_{|x| \leqslant 1}\right) \nu(d x) \\
& =i u \int_{|x| \geqslant 1} x \nu(d x)+C \int_{0}^{\infty}\left(e^{i u x}-1-i u x\right) \frac{\lambda^{(\alpha+1) / 2} K_{(\alpha+1) / 2}(\lambda x)}{x^{(\alpha+1) / 2}} d x \\
& \quad+C \int_{-\infty}^{0}\left(e^{i u x}-1-i u x\right) \frac{e^{-\lambda|x|}}{|x|^{\alpha+1}} d x \\
& =i u \int_{|x| \geqslant 1} x \nu(d x)+G_{M}(i u ; \alpha, \lambda, C)+G_{C}(-i u ; \alpha, \lambda, C) .
\end{aligned}
$$

For more details see Kim et al. [113] and Carr et al. [5]. The Lévy-Khintchine formula completes the proof.

Proposition 3.1. Let $X \sim A C M T S(\alpha, C, \lambda, \mu)$. Then the Laplace transform $E\left[e^{\theta X}\right]$ is finite for all $\theta \in[-\lambda, \lambda]$. Moreover, the explicit formula of the Laplace transform is given by

$$
E\left[e^{\theta X}\right]=\exp \left(\theta \mu+G_{M}(\theta ; \alpha, \lambda, C)+G_{C}(-\theta ; \alpha, \lambda, C)\right) .
$$




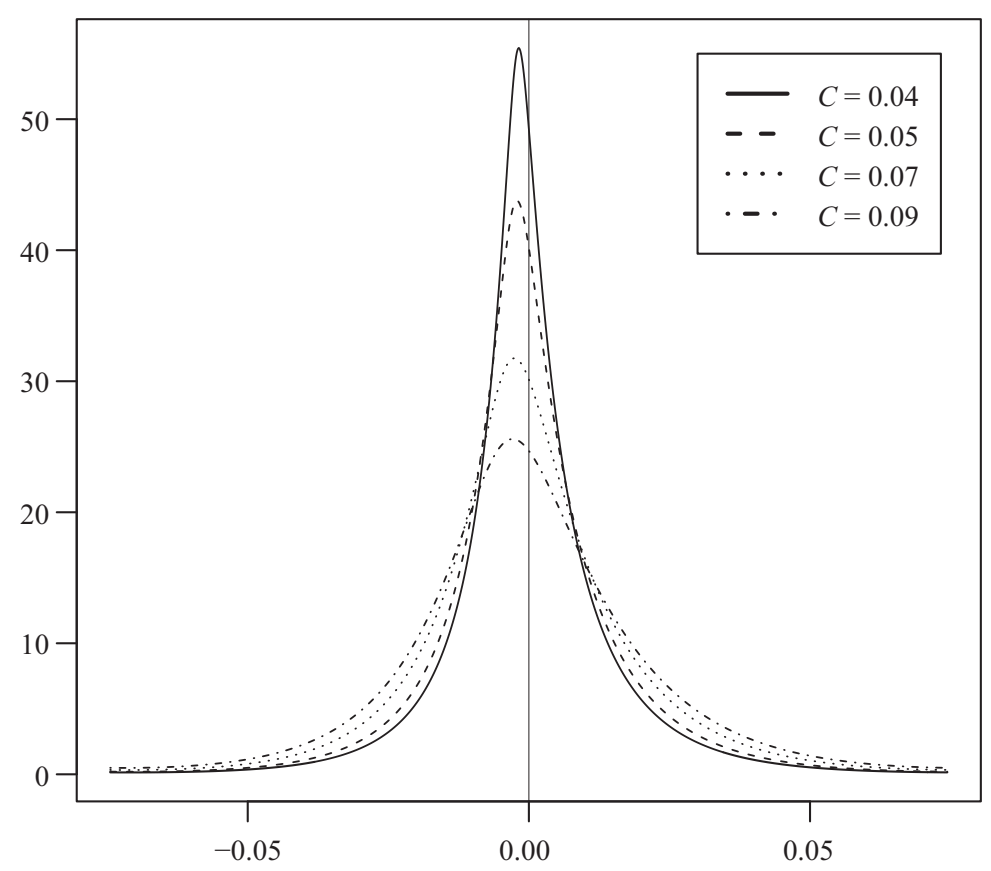

Figure 2. Probability density of the ACMTS distributions: dependence on $C$. Parameters: $\mu=0, \alpha=0.5, \lambda=55$.

Proposition 3.2. Let $X \sim A C M T S(\alpha, C, \lambda, \mu)$. The cumulants $c_{n}$ of $X$ are given by $c_{1}(X)=\mu$ and

$c_{n}(X)=\left\{\begin{array}{l}2^{n-(\alpha+3) / 2}\left(\frac{n-1}{2}\right) ! C \Gamma\left(\frac{n-\alpha}{2}\right) \lambda^{\alpha-n}-C \Gamma(n-\alpha) \lambda^{\alpha-n}, n=3,5, \ldots, \\ 2^{-(\alpha+3) / 2} \sqrt{\pi} \frac{n !}{(n / 2) !} C \Gamma\left(\frac{n-\alpha}{2}\right) \lambda^{\alpha-n}+C \Gamma(n-\alpha) \lambda^{\alpha-n}, n=2,4, \ldots\end{array}\right.$

Moreover, using the cumulants, we obtain the mean, variance, skewness, and excess kurtosis:

$$
\begin{gathered}
E(X)=c_{1}(X)=\mu, \\
\operatorname{Var}(X)=c_{2}(X)=2^{-(1+\alpha) / 2} \sqrt{\pi} C \Gamma\left(\frac{2-\alpha}{2}\right) \lambda^{\alpha-2}+C \Gamma(2-\alpha) \lambda^{\alpha-2}, \\
s(X)=\frac{c_{3}(X)}{c_{2}(X)^{3 / 2}}=\frac{\lambda^{-\alpha / 2}}{\sqrt{C}} \frac{2^{(3-\alpha) / 2} \Gamma\left(\frac{3-\alpha}{2}\right)-\Gamma(3-\alpha)}{\left(2^{-(1+\alpha) / 2} \sqrt{\pi} \Gamma\left(\frac{2-\alpha}{2}\right)+\Gamma(2-\alpha)\right)^{3 / 2}}, \\
k(X)=\frac{c_{4}(X)}{c_{2}(X)^{2}}=\frac{\lambda^{-\alpha-2}}{C} \frac{2^{(1-\alpha) / 2} \cdot 3 \sqrt{\pi} \Gamma\left(\frac{4-\alpha}{2}\right)+\Gamma(4-\alpha)}{\left(2^{-(1+\alpha) / 2} \sqrt{\pi} \Gamma\left(\frac{2-\alpha}{2}\right)+\Gamma(2-\alpha)\right)^{2}} .
\end{gathered}
$$

It is easy to see that $s(X)>0$, so the ACMTS distribution is always skewed to the right. The parameter $\lambda$ controls the rate of decay on tail. If $\lambda$ increases, then 


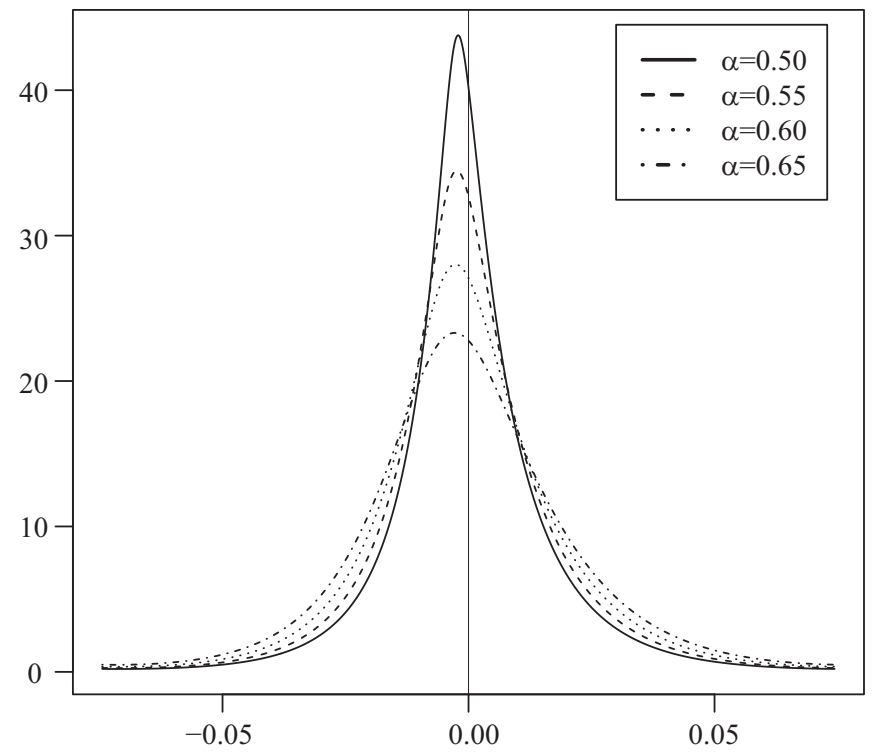

FIGURE 3. Probability density of the ACMTS distributions: dependence on $\alpha$. Parameters: $\mu=0, C=0.05, \lambda=55$.

the skewness of the distribution decreases. Figure $\mathbb{U}$ shows the effect of $\lambda$. The parameter $C$ determines the arrival rate of jumps for a given size. Figure $\square$ shows the effect of $C$. The ACMTS is infinitely divisible. The Lévy process generated by this distribution is called the ACMTS process with parameters $(\alpha, C, \lambda, \mu)$. This process has finite variation if $\alpha \in(0,1)$ and infinite variation if $\alpha \in(1,2)$. Figure 3 shows the effect of $\alpha$.

\subsection{The AMCTS distribution.}

DEFINITION 3.2. An infinitely divisible distribution is called an AMCTS distribution with parameters $(\alpha, C, \lambda, \mu)$ if its Lévy triplet $(A, \nu, \gamma)$ is given by $A=0$ and

$$
\nu(d x)=\left(C \frac{\lambda^{(\alpha+1) / 2} K_{(\alpha+1) / 2}(\lambda|x|)}{|x|^{(\alpha+1) / 2}} 1_{x<0}+C \frac{e^{-\lambda x}}{x^{\alpha+1}} 1_{x>0}\right) d x,
$$

and

$$
\gamma=\mu-\int_{|x|>1} x \nu(d x)
$$

where $C, \lambda>0, \mu \in \mathbb{R}$ and $\alpha \in(0,2) \backslash\{1\}$.

If a random variable $X$ follows the AMCTS distribution, then we write $X \sim$ $A M C T S(\alpha, C, \lambda, \mu)$. The Lévy measure of the AMCTS distribution is given by

$$
\nu(d r, d u)=r^{-\alpha-1} q(r, u) d r \sigma(d u),
$$




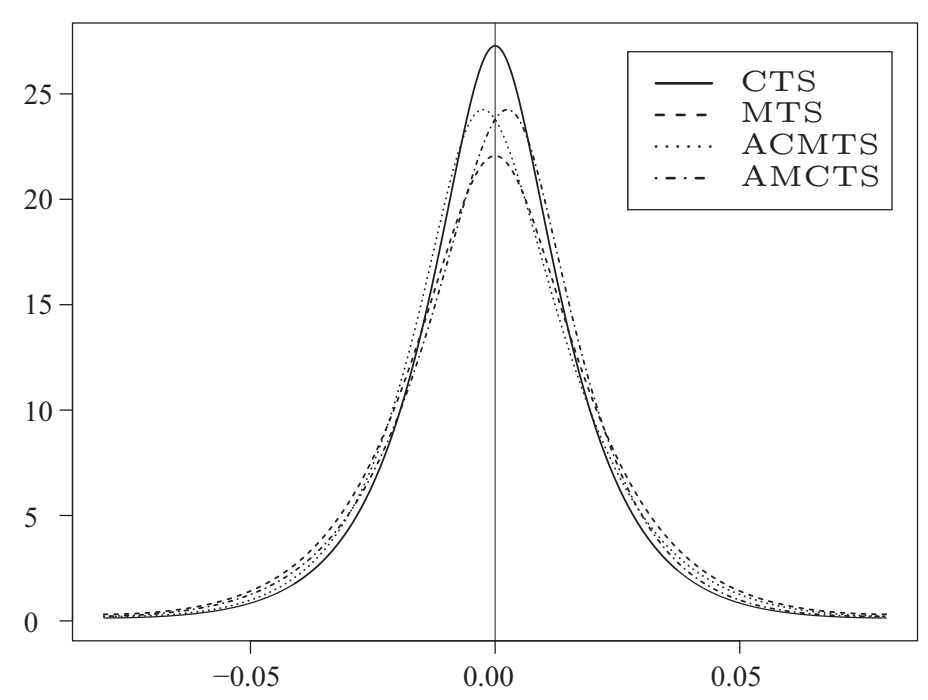

FIGURE 4. Probability density of the CTS, MTS, ACMTS and AMCTS distributions. Parameters: $\mu=0, C=0.2, \alpha=0.4$ and $\lambda_{-}=\lambda_{+}=\lambda=75$.

where $q(r,-1)=(\lambda r)^{(\alpha+1) / 2} K_{(\alpha+1) / 2}(\lambda r), q(r, 1)=e^{-\lambda r}, \sigma(1)=\sigma(-1)=C$. The AMCTS distributions are not included in the class of TS distributions but belong to the class of GTS distributions. The AMCTS distribution is similar to the ACMTS distribution with the difference that the right and left tails of the AMCTS distribution are replaced with the left and right tails of the ACMTS distribution, respectively; see Figure 4 . Indeed, if $X$ has the ACMTS distribution with parameters $\alpha, C, \lambda$ and $\mu$, then $Y=-X$ has the AMCTS distribution with parameters $\alpha, C, \lambda$ and $-\mu$. It is obvious that $E(Y)=-E(X), \operatorname{Var}(Y)=\operatorname{Var}(X), s(Y)=$ $-s(X), k(Y)=k(X)$ and the characteristic function of $Y$ is given by $\phi_{Y}(u)=$ $\exp \left(i u \mu+G_{C}(i u ; \alpha, \lambda, C)+G_{M}(-i u ; \alpha, \lambda, C)\right)$. The Laplace transform $E\left[e^{\theta Y}\right]$ is finite for all $\theta \in[-\lambda, \lambda]$ and the explicit formula of the Laplace transform is given by $E\left[e^{\theta Y}\right]=\exp \left(\theta \mu+G_{C}(\theta ; \alpha, \lambda, C)+G_{M}(-\theta ; \alpha, \lambda, C)\right)$. The AMCTS distribution is infinitely divisible. The Lévy process generated by this distribution is called the AMCTS process with parameters $(\alpha, C, \lambda, \mu)$. This process has finite variation if $\alpha \in(0,1)$ and infinite variation if $\alpha \in(1,2)$.

\section{ASYMMETRICALLY TEMPERED STABLE MARKET MODEL}

Let $\Omega$ be the set of all càdlàg functions on $[0, T]$ and $\left(X_{t}\right)_{t \in[0, T]}$ be a Lévy process on a sample space $(\Omega, \mathbb{P})$. In the market, if the stock price is given by the random variable $S_{t}=S_{0} e^{X_{t}}, t \in[0, T]$, for some initial value of the stock price $S_{0}>0$, then we say that the stock price follows an exponential model. Let us denote the risk-free interest rate by $r>0$. The discounted stock price $\tilde{S}_{t}$ of $S_{t}$ is given by $\tilde{S}_{t}=e^{-r t} S_{t}, t \in[0, T]$. The process $\left(X_{t}\right)_{t \in[0, T]}$ is called the driving pro- 
cess of $\left(S_{t}\right)_{t \in[0, T]}$. The driving process $\left(X_{t}\right)_{t \in[0, T]}$ is completely described by the market measure $\mathbb{P}$. If $\left(X_{t}\right)_{t \in[0, T]}$ is a Lévy process under the measure $\mathbb{P}$, we say that the stock price process follows the exponential Lévy model. In particular, if the driving process is the asymmetrically tempered stable process, the exponential Lévy model is referred to as the exponential asymmetrically tempered stable model. A probability measure $\mathbb{Q}$ equivalent to $\mathbb{P}$ is called an equivalent martingale measure (EMM) of $\mathbb{P}$ if the discounted stock price process is a $\mathbb{Q}$-martingale (see Lewis [118]), that is, $E^{\mathbb{Q}}\left[S_{t}\right]=e^{r t} S_{0}$.

Now, we review a general result of equivalence measures presented by Sato [21] and apply it to the asymmetrically tempered stable distribution. The following theorem is a particular case of Theorem 33.1 in Sato [21]:

THEOREM 4.1. Let $(X ; \mathbb{P})$ and $(X ; \mathbb{Q})$ be two infinitely divisible random variables on $\mathbb{R}$ with Lévy triplets $(A, \nu, \gamma)$ and $(\tilde{A}, \tilde{\nu}, \tilde{\gamma})$, respectively. Then $\mathbb{P}$ and $\mathbb{Q}$ are equivalent if and only if

$$
\begin{gathered}
A=\tilde{A}, \\
\int_{-\infty}^{\infty}\left(e^{\psi(x) / 2}-1\right)^{2} \nu(d x)<\infty,
\end{gathered}
$$

where $\psi(x)=\ln (\nu(x) / \tilde{\nu}(x))$. If $A=0$, then

$$
\tilde{\gamma}-\gamma=\int_{|x| \leqslant 1} x(\tilde{\nu}-\nu)(d x) .
$$

4.1. Exponential ACMTS model. Since ACMTS distributions are infinitely divisible, we can apply Theorem 4.$]$ to obtain the change of measure.

Proposition 4.1. Let $(X ; \mathbb{P})$ and $(X ; \mathbb{Q})$ be two ACMTS distributed random variables on $\mathbb{R}$ with parameters $(\alpha, C, \lambda, \mu)$ and $(\tilde{\alpha}, \tilde{C}, \tilde{\lambda}, \tilde{\mu})$, respectively. Then $\mathbb{P}$ and $\mathbb{Q}$ are equivalent if and only if $C=\tilde{C}, \alpha=\tilde{\alpha}$ and $\mu=\tilde{\mu}$.

Proof. Let $(0, \nu, \gamma)$ and $(0, \tilde{\nu}, \tilde{\gamma})$ be Lévy triplets of $\left(X_{t}, \mathbb{P}\right)$ and $\left(X_{t}, \mathbb{Q}\right)$, respectively. Since the diffusion coefficients of ACMTS processes are zero, (4.I) holds. From the definition of Lévy measures $\tilde{\nu}$ and $\nu$ it follows that $\psi(x)$ in (4.2) is equal to $\psi(x)=\psi_{-}(x)+\psi_{+}(x)$, where

$$
\psi_{-}(x)=\left(\ln \left(\frac{\tilde{C}|x|^{-\tilde{\alpha}}}{C|x|^{-\alpha}}\right)+x(\gamma-\tilde{\gamma})\right) 1_{x<0}
$$

and

$$
\psi_{+}(x)=\ln \left(\frac{\tilde{\lambda}^{\tilde{\alpha} / 2+1 / 2} K_{\tilde{\alpha} / 2}(\tilde{\lambda} x) x^{\alpha / 2+1 / 2}}{\lambda^{\alpha / 2+1 / 2} K_{(\alpha+1) / 2}(\lambda x) x^{\tilde{\alpha} / 2+1 / 2}}\right) 1_{x>0} .
$$

We can prove that $\int_{-\infty}^{0}\left(e^{\psi_{-}(x) / 2}-1\right) d x<\infty$ if and only if $\alpha=\tilde{\alpha}$ and $C=\tilde{C}$ (see Kim et al. [I]]), and $\int_{0}^{\infty}\left(e^{\psi_{+}(x) / 2}-1\right) d x<\infty$ if and only if $\alpha=\tilde{\alpha}$ and 
$C=\tilde{C}$. Thus, the condition (4.2) holds if and only if $\alpha=\tilde{\alpha}$ and $C=\tilde{C}$. It is clear from Definitions 2.4 and 2.5 that (4.3) holds if and only if $\tilde{\mu}=\mu$.

The ACMTS model is defined under the continuous-time market as follows. For convenience, let us put

$$
\psi_{A C M T S}^{0}(u, \alpha, C, \lambda)=G_{M}(u ; \alpha, \lambda, C)+G_{C}(-u ; \alpha, \lambda, C) .
$$

Definition 4.1. Let $C>0, \lambda>1, \alpha \in(0,2) \backslash\{1\}$ and $\mu \in \mathbb{R}$. In the continuous-time market, if the driving process $\left(X_{t}\right)_{t \in[0, T]}$ of $\left(S_{t}\right)_{t \in[0, T]}$ is an ACMTS process with parameters $(\alpha, C, \lambda, m)$ and $m=\mu-\psi_{A C M T S}^{0}(1, \alpha, C, \lambda)$, then $\left(S_{t}\right)_{t \in[0, T]}$ is called the ACMTS stock price process with parameters $(\alpha, C, \lambda, \mu)$ and we say that the stock price process follows the ACMTS model.

The function $\psi_{A C M T S}^{0}(1, \alpha, C, \lambda)$ is well defined with the condition $\lambda>1$, and hence $E\left[S_{t}\right]=S_{0} e^{\mu t}$. If we apply Proposition 4.] to the ACMTS model, we obtain the following theorem.

THEOREM 4.2 (EMM conditions for the ACMTS model). Assume that the process $\left(S_{t}\right)_{t \in[0, T]}$ is the ACMTS stock price process with parameters $(\alpha, C, \lambda, \mu)$ under the market measure $\mathbb{P}$, and with parameters $(\tilde{\alpha}, \tilde{C}, \tilde{\lambda}, r)$ under a measure $\mathbb{Q}$. Then $\mathbb{Q}$ is an EMM of $\mathbb{P}$ if and only if $C=\tilde{C}, \alpha=\tilde{\alpha}$ and

$$
r-\psi_{A C M T S}^{0}(1, \tilde{\alpha}, \tilde{C}, \tilde{\lambda})=\mu-\psi_{A C M T S}^{0}(1, \alpha, C, \lambda) .
$$

\section{ESTIMATION OF MARKET PARAMETERS}

In this section, we report the maximum likelihood estimation of the exponential CST, MTS, ACMTS and AMCTS models. We use historical prices of the Standard and Poor's (S\&P) 500 Index for our illustration. The time series of the closing prices are from February 6, 1995 to February 6, 2015.

For the assessment of the goodness-of-fit, we use the Kolmogorov-Smirnov (KS) test. The KS statistic is defined as follows:

$$
K S=\sup _{x_{i}}\left|F\left(x_{i}\right)-\hat{F}\left(x_{i}\right)\right|,
$$

where $F$ is the cumulative distribution function and $\hat{F}$ is the empirical cumulative distribution function for a given observation $\left\{x_{i}\right\}$. Table 1 provides the results. According to this table, since the number of parameters of the CTS and MTS distributions is greater than that of the ACMTS and AMCTS distributions, the $p$-values of the CTS and MTS models are larger than those of the ACMTS and AMCTS models. On the other hand, the skewness log-returns data is -0.2435 , the ACMTS distribution is always skewed to the right and the AMCTS distribution is always skewed to the left, thus we expect that $p$-values of the AMCTS model are larger than those of the ACMTS model, which is confirmed by Table 1. 
TABLE 1. Market parameters for the S\&P 500 Index.

\begin{tabular}{|ccccccc|}
\hline Model & \multicolumn{7}{c|}{ Parameters } \\
\hline CTS & $\mu$ & $\alpha$ & $C$ & $\lambda_{+}$ & $\lambda_{-}$ & $K S$ ( $p$-value $)$ \\
& 0.00027 & 0.3581 & 0.1044 & 78.7269 & 72.9162 & $0.0108(0.8769)$ \\
MTS & $\mu$ & $\alpha$ & $C$ & $\lambda_{+}$ & $\lambda_{-}$ & $K S$ ( $p$-value $)$ \\
& 0.00027 & 0.7442 & 0.0113 & 80.4786 & 64.8835 & 0.0106 (0.8979) \\
& & & & & & \\
ACMTS & $\mu$ & $\alpha$ & $C$ & $\lambda$ & & $K S$ ( $p$-value) \\
& 0.0013 & 0.5577 & 0.03515 & 77.2954 & & $0.0248(0.0912)$ \\
& & & & & & \\
AMCTS & $\mu$ & $\alpha$ & $C$ & $\lambda$ & & $K S(p$-value $)$ \\
& 0.0001 & 0.5168 & 0.0462 & 84.8664 & & $0.0125(0.7835)$ \\
\hline
\end{tabular}

\section{CONCLUSION}

In this paper, we introduce two new tempered stable distributions named the ACMTS and AMCTS distributions that are obtained by combining CTS and MTS distributions. These distributions are not in the class of the tempered stable distributions introduced by Rosiński [19] but they are in the class of the generalized tempered stable distributions defined by Rosiński and Sinclair [20]. For the ACMTS distribution the left tail is similar to that of the CTS distribution and the right tail is similar to that of the MTS distribution. The AMCTS distribution is the opposite, in which the left tail of distribution is similar to that of the MTS distribution and the right tail is similar to that of the CTS distribution. Consequently, the ACMTS and AMCTS models can be more realistic than the CTS and MTS models. We fit S\&P 500 Index returns time series from February 6, 1995 to February 6, 2015. The KS tests do not reject the CTS, MTS and AMCTS distributions. Although the AMCTS distribution has one parameter less, the $p$-values of the KS statistic for the AMCTS distribution are similar to those of the CTS and MTS distributions.

In this paper, we use the CTS and MTS distributions to construct the ATS distribution. However, other tempered stable distributions can be used to obtain some types of the ATS distributions which are suitable for different situations - for example, asymmetrically CTS and RDTS distributions and asymmetrically RDTS and MTS distributions.

\section{REFERENCES}

[1] O. E. Barndorff-Nielsen and S. Z. Levendorskiì, Feller processes of normal inverse Gaussian type, Quant. Finance 1 (3) (2001), pp. 318-331.

[2] M. L. Bianchi, S. T. Rachev, Y. S. Kim, and F. J. Fabozzi, Tempered infinitely divisible distributions and processes, Theory Probab. Appl. 55 (1) (2010), pp. 59-86. 
[3] F. Black and M. Scholes, The pricing of options and corporate liabilities, J. Political Economy 81 (3) (1973), pp. 637-654.

[4] S. I. Boyarchenko and S. Z. Levendorski , Option pricing for truncated Lévy processes, Int. J. Theor. Appl. Finance 3 (3) (2000), pp. 549-552.

[5] P. Carr, H. Geman, D. Madan, and M. Yor, The fine structure of asset returns: An empirical investigation, J. Business 75 (2) (2002), pp. 305-332.

[6] R. Cont and P. Tankov, Financial Modelling with Jump Processes, Chapman\&Hall/CRC, Boca Raton 2004.

[7] H. U. Gerber and E. S. W. Shiu, Option pricing by Esscher transforms (with discussions), Trans. Soc. Actuar. 46 (1994), pp. 99-191.

[8] H. U. Gerber and E. S. W. Shiu, Actuarial bridges to dynamic hedging and option pricing, Insurance Math. Econom. 18 (3) (1996), pp. 183-218.

[9] S. R. Hurst, E. Platen, and S. T. Rachev, Option pricing for a logstable asset price model, Math. Comput. Modelling 29 (1999), pp. 105-119.

[10] Y. S. Kim, S. T. Rachev, M. L. Bianchi, and F. J. Fabozzi, A new tempered stable distribution and its application to finance, in: Risk Assessment: Decisions in Banking and Finance, G. Bol, S. T. Rachev, and R.Wuerth (Eds.), Physika Verlag, Springer, 2008, pp. 77-119.

[11] Y. S. Kim, S. T. Rachev, M. L. Bianchi, and F. J. Fabozzi, Tempered stable and tempered infinitely divisible GARCH models, Technical Report, University of Karlsruhe and KIT, 2009.

[12] Y. S. Kim, S. T. Rachev, M. L. Bianchi, and F. J. Fabozzi, Tempered stable and tempered infinitely divisible GARCH models, J. Bank. Finance 34 (9) (2010), pp. 2096-2109.

[13] Y. S. Kim, S. T. Rachev, D. M. Chung, and M. L. Bianchi, The modified tempered stable distribution, GARCH models and option pricing, Technical Report, Chair of Econometrics, Statistics and Mathematical Finance, School of Economics and Business Engineering, University of Karlsruhe, 2008.

[14] Y. S. Kim, S. T. Rachev, D. M. Chung, and M. L. Bianchi, A modified tempered stable distribution with volatility clustering, in: New Developments in Financial Modelling, J. O. Soares, J. P. Pina, and M. Catalaõ-Lopes (Eds.), Cambridge Scholars Publishing, Newcastle upon Tyne 2008, pp. 344-365.

[15] Y. S. Kim, S. T. Rachev, D. M. Chung, and M. L. Bianchi, The modified tempered stable distribution, GARCH models and option pricing, Probab. Math. Statist. 29 (1) (2009), pp. 91-117.

[16] I. Koponen, Analytic approach to the problem of convergence of truncated Lévy flights towards the Gaussian stochastic process, Phys. Rev. E 52 (1995), pp. 1197-1199.

[17] U. Küchlera and S. Tappe, Tempered stable distributions and processes, Stochastic Process. Appl. 123 (2013), pp. 4256-4293.

[18] A. L. Lew is, A simple option formula for general jump-diffusion and other exponential Lévy processes, SSRN Electronic Journal, May 2002.

[19] J. Rosiński, Tempering stable processes, Stochastic Process. Appl. 117 (6) (2007), pp. 677707.

[20] J. Rosiński and J. L. Sinclair, Generalized tempered stable processes, in: Stability in Probability, J. K. Misiewicz (Ed.), Banach Center Publ., Vol. 90, Warsaw 2010, pp. 153-170.

[21] K. Sato, Lévy Processes and Infinitely Divisible Distributions, Cambridge University Press, Cambridge 1999.

A. Arefi

Department of Statistics

Allameh Tabataba'i University, Iran

E-mail: arefi921@atu.ac.ir
R. Pourtaheri

Department of Statistics Allameh Tabataba'i University, Iran

Received on 3.8.2015;

revised version on 19.9.2017 